\title{
The Surgeon's Contribution to Image-Guided Oncology
}

\author{
Christoph Benckert ${ }^{\mathrm{a}, \mathrm{b}} \quad$ Christiane Bruns $^{\mathrm{a}, \mathrm{b}}$ \\ ${ }^{a}$ Deutsche Akademie für Mikrotherapie (DAfMT)/International School of Image-Guided Interventions, Magdeburg, Germany

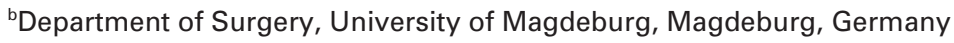

\section{Keywords}

Laparoscopic liver surgery · Image guidance . Intraoperative ultrasound - IOUS . Three-dimensional computer-assisted surgical planning - 3D-CASP . Magnetic resonance imaging . Computed tomography . Fluorescent imaging · Augmented reality · Hybrid operating room

\section{Summary}

Background: Advances in surgical and in imaging technology permit the performance of complex tumour resections in a safe and oncologically correct manner. To date, this has mainly implicated refined preoperative imaging methods, such as three-dimensional computer-assisted planning (3DCASP). With the advent of modern hybrid operating rooms, intraoperative imaging has spread and various techniques of intraoperative image guidance have been developed. Methods: We review recent advances in intraoperative image guidance. We also delineate the role of intraoperative imaging techniques such as intraoperative ultrasound and computed tomography for real-time image guidance in laparoscopic liver surgery. Results: Our review shows that advances in intraoperative imaging accompany the increasing use of laparoscopic approaches in visceral surgery. For the liver surgeon working laparoscopically, the loss of tactile sensation and the complex three-dimensional anatomy of the human liver make 3D-imaging techniques and intraoperative image guidance indispensable. We describe the role of 3D-CASP in preoperative surgical planning in liver surgery. Conclusion: An innovative imaging strategy for identifying liver segments during laparoscopic liver surgery by applying a fluorescent imaging method is proposed.

\section{Schlüsselwörter \\ Laparoskopische Leberchirurgie · Bildführung · Intraopera- tiver Ultraschall · IOUS . Dreidimensionale computerassi- stierte chirurgische Planung - 3D-CASP . Magnetresonanz- tomographie - Computertomographie - Fluoreszenzbild- gebung · Erweiterte Realität · Hybridoperationssaal}

\section{Zusammenfassung}

Hintergrund: Fortschritte in den chirurgischen und bildgebenden Verfahren erlauben zunehmend komplexe onkologische Tumorresektionen in einer für den Patienten sicheren, d.h. onkologisch korrekten und damit lebensverlängernden Art und Weise. Moderne, präoperative bildgebende Verfahren, wie die dreidimensionale computerassistierte chirurgische Planung (3D-CASP), führen zu einer besseren Einschätzung der operativen Risiken beispielsweise in der Leberchirurgie. Mit der Einführung und Verbreitung von modernen Hybridoperationssälen hat die intraoperative Bildgebung vor allem in der Gefäß- und Neurochirurgie Verbreitung gefunden, und verschiedene Anwendungen für intraoperative Bildgebungen sind entwickelt worden. Methoden: Wir geben eine Übersicht über innovative Anwendungen der intraoperativen Bildgebung in der Leberchirurgie. Hierbei wird die Rolle des intraoperativen Ultraschalls und der intraoperativen Computertomographie für die Echtzeitbildgebung während laparoskopischen Leberoperationen skizziert. Ergebnisse: Wir zeigen, dass Fortschritte im Bereich der intraoperativen Bildgebung mit einer vermehrten Anwendung laparoskopischer Verfahren in der Leberchirurgie einhergehen. Wir beschreiben die Rolle von 3D-CASP für die Planung von Leberresektionen und skizzieren Möglichkeiten der Bildführung während laparoskopischen Leberoperationen mittels Überlagerung von 3D-Rekonstruktionen und Daten einer intraoperativen Computertomographie auf dem Videomonitor. Schlussfolgerung: Ein innovativer Ansatz zur Lebersegmentmarkierung während der laparoskopischen Leberchirurgie mithilfe von Fluoreszenzbildgebung wird vorgestellt.

\section{KARGER \\ Fax +497614520714 \\ Information@Karger.com}

www.karger.com
(C) 2014 S. Karger GmbH, Freiburg

1662-6664/14/0304-0232\$39.50/0

Accessible online at:

www.karger.com/vim 


\section{Introduction}

Innovative multidisciplinary approaches consisting of chemotherapeutic, (interventional) radiological, and surgical regimens have led to a better long-term survival of patients with visceral malignancies. In metastatic bilobal colorectal liver disease, for example, oncosurgery approaches in multidisciplinary teams may lead to complete tumour resection in patients initially classified as non-resectable [1]. Against this background, surgeons are being confronted with an increasing demand for more complex and more frequent surgical tumour resections in patients with extended tumour spread.

Advances in surgical techniques, research, and perioperative medicine have led to reduced morbidity and mortality in patients undergoing major abdominal surgery. From the surgeon's point of view, however, it will be an increasing challenge to find the appropriate balance between oncological correctness and safety in an extended tumour resection. Preand intraoperative image guidance will be an essential tool to ensure the best possible oncological result without sacrificing the patients' safety.

With the increasing implementation of laparoscopic approaches and the associated loss of tactile sensation, intraoperative image guidance will be an important tool for selecting the correct oncological procedure. Especially in oncological liver surgery, image guidance is indispensable. Ideally, image guidance should replace tactile sensation in guiding the liver surgeon. This article focuses on various aspects of image guidance in oncological liver surgery.

\section{Preoperative Image Guidance and Surgical Planning}

The role of preoperative imaging in oncological liver surgery has shifted from pure detection of malignant lesions and the vague assessment of resectability towards an exact definition of the surgical strategy based on computed tomography (CT) and magnetic resonance imaging (MRI). Impelled by the surgeon's wish to reduce the patient's exposure to risks entailed by liver surgery (i.e. intraoperative bleeding, postoperative liver failure, bile leakage, etc.), and in order to optimise individual surgical planning, three-dimensional computer-assisted surgical planning (3D-CASP) has been established as a valuable additional modality. Particularly for liver surgeons, 3D imaging is appealing because of the complex 3D anatomy of the human liver.

The optimum surgical strategy includes not only the determination of the exact parenchymal transection course and the planning of vascular or biliary reconstructions but also the choice between a laparoscopic or an open approach. For complex liver resections (extended right or left hepatectomies, trisectionectomies, repeated liver resections) an exact knowledge of the intrahepatic vascular (arterial, portal, and hepatic venous) and biliary anatomy, which varies in the liver to a sig-

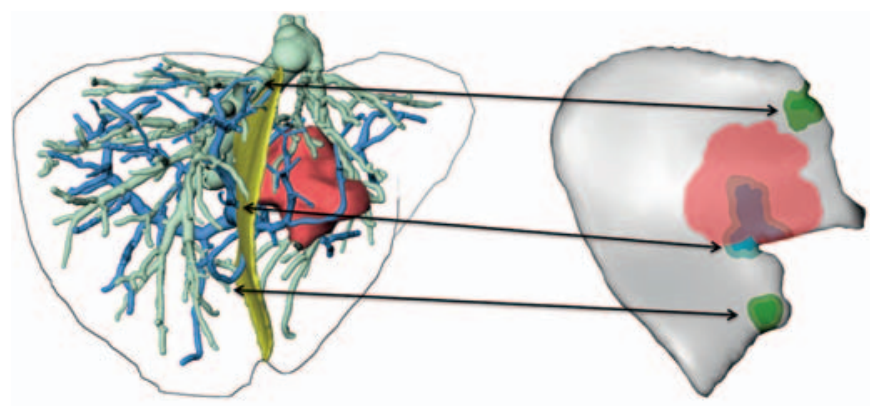

Fig. 1. Patient-individual $3 \mathrm{D}$ model of a liver including a virtual resection surface (left). Risk structures close to the planned resection surface are visualized on a colour-coded risk map (right). Image courtesy of Christian Hansen (University of Magdeburg) and Fraunhofer MEVIS, Bremen, Germany.

nificant extent, is essential; this can reduce the incidence of postoperative complications such as biliary leakage, segmental devascularisation, or impaired venous drainage and the resulting loss of functional capacity of the remnant liver. Radtke et al. [2] have described a series of 202 consecutive hepatectomies in which information gathered from additional 3DCASP, in comparison with conventional two-dimensional (2D) CT data, changed the surgical strategy in $33 \%$ of the patients. The preoperative switch in surgical approach included i) expansion of the resection, ii) planning of intrahepatic vascular reconstruction, and iii) complete change in the surgical strategy. In particular, 3D-CASP has been shown to be useful in the planning of extended left hepatectomies and repeated liver resections. Figure 1 shows a 3D model of a liver highlighting risk structures close to the planned parenchymal transection line.

Within emerging multidisciplinary treatment modalities, neoadjuvant chemotherapy regimens are being developed as effective therapeutic tools. 'Complete' clinical response rates are seen more frequently, leading to vanishing lesions, which can neither be seen by intraoperative ultrasound (IOUS) nor be palpated. Owing to the high rate of intrahepatic recurrence, a surgical resection should still be performed, and this needs exact preoperative image guidance [3].

$3 \mathrm{D}$ modelling requires powerful software packages in order to generate individual liver models that reflect the complex anatomical structure of each patient's liver. The requirements of the raw data for 3D-CASP have been outlined in a review article by Chopra et al. [4]. Until now, CT datasets have generally been used for 3D modelling; however, MRI datasets can be used as well and, looking ahead, 3D modelling from MR images should be implemented as a standard procedure due to the low exposure to radiation that it entails. In support of the extensive use of MRI, it is not only the patients' nonexposure to radiation that counts; intraoperative MRI also appears to be the only acceptable modality for surgeons and operating room personnel, with regard to both imaging quality and radiation exposure. These factors will increase in im- 
portance against the background of the fact that intraoperative imaging, in so-called hybrid operating theatres, is becoming more and more important and probably will become the standard - not only in neurosurgery but in operating theatres in general - within the next 15 years.

Assuming that the source datasets will be generated by MRI in the future, the requirements for the software packages will be even more challenging since MRI data of the liver are less uniform, owing to multiple varying sequence parameters [4]. Beside the avoidance of radiation exposure, an additional important advantage of MRI is its higher sensitivity and specificity in detecting small hepatic lesions when compared with CT imaging [5]. Even in open liver surgery, small hepatic lesions are not always palpable, but for laparoscopic liver surgeons these lesions are never palpable and a reliable visualisation (i.e. by means of MRI) is indispensable. Considering the visualisation of hepatic vessels, portal vessels are visualised even better by contrast-enhanced CT imaging; however, angiomagnetic imaging should outweigh this disadvantage in the future.

\section{Intraoperative Image Guidance}

\section{Ultrasound}

IOUS as the mainstay of image guidance during liver surgery has been established for almost 30 years. It was first described by Makuuchi et al. [6]. Initially, IOUS mainly provided the opportunity for the liver surgeon to detect hepatic vessels and to determine the transection line. Identification of the middle hepatic vein, which varies in between individuals to quite a large extent, is an essential step in determining the parenchymal transection line for an anatomic right hepatectomy. Up to now, IOUS has been found to be the most reliable imaging modality in detecting (additional) colorectal tumour manifestations. In a prospective randomised study comparing the diagnostic performance of CT scan, MR liver, PET(positron emission tomography)-CT, and IOUS, IOUS showed the highest concordance with postoperative histopathology for the number of liver metastases [7]. The importance of this approach for the patient is underlined by the fact that IOUS findings may lead to a change in the surgical strategy for $7.5-35 \%$ of patients $[7,8]$.

\section{Laparoscopic Liver Surgery}

Minimally invasive liver surgery is still technically challenging, and therefore the laparoscopic approach is not as widespread in liver surgery as in colorectal surgery. Bleeding within the liver parenchyma is more difficult to control than in surgery of the lower abdomen and results in a high rate of conversion to open surgery. In addition, liver surgeons are usually trained in open surgery, and experienced laparoscopic surgeons are commonly not trained in liver surgery. However, there is an increasing number of publications describing lapa- roscopic hepatectomy as a safe procedure, with comparable oncological results [9-11]. The known advances of laparoscopic surgery such as reduced bleeding, lower morbidity, and reduced hospital stay are also found in minimally invasive liver resections, particularly in patients with liver cirrhosis [12]. Even though major extended hepatectomies are feasible, there is a consensus that the laparoscopic approach should be the standard only for lesions smaller than $5 \mathrm{~cm}$ in the left lateral and anteroinferior liver segments (segments 2, 3, 5, and 6) [13]. In addition, it seems obvious that the lack of tactile sensation and the more complex anatomical orientation associated with laparoscopic liver surgery should be compensated for by image guidance.

Since prevention of vascular injury is fundamental, precise knowledge of the vascular anatomy is necessary. This can be achieved by exact preoperative imaging through $\mathrm{CT}$ and MR datasets or by intraoperative guidance - in real time - during surgery. For an easy application of image guidance during laparoscopic liver surgery it is advisable to use a 'picture in picture' mode on the laparoscopic monitor, allowing one to see both laparoscopic and preoperatively acquired CT or MR or real-time imaging datasets. Real-time image guidance in laparoscopic liver surgery has up to now been based on IOUS. In experienced hands, IOUS can provide adequate guidance for anatomic (i.e. segmental) transection lines. However, deeply located liver tumours cannot be detected by IOUS, which is a strong limitation of this modality. In order to overcome the limitation of IOUS, various research groups have worked on navigation systems for laparoscopic liver surgery using optical tracking systems combined with preoperative CT and MRI datasets [4]. Intraoperative navigation to date means visualising preoperatively acquired, computer-modelled datasets in relation to the real patient and the surgical instruments. In clinical practice, navigation systems with proven value for the liver surgeon are not yet applicable. The rapid spread of hybrid operating rooms equipped with imaging systems allowing intraoperative CT imaging, e.g. by conebeam computed tomography (CBCT), facilitates the intraoperative detection of lesions located deeply in the liver parenchyma and the identification of vascular structures during laparoscopic liver surgery.

\section{Augmented Reality Guidance during Laparoscopic Liver Surgery}

Augmented reality (AR) in the context of laparoscopic surgery is defined as the overlay of computer-generated images on real-world structures seen on the laparoscopic monitor. In a recent publication by Kenngott et al. [14], a promising approach of real-time image guidance in laparoscopic liver surgery combining an AR software guidance system with intraoperative CBCT was described. In the setting of a modern hybrid operating theatre, intraoperative fluoroscopy and CT images were overlaid with a 3D model of the liver, providing image guidance by AR and allowing the surgeon to find a pre- 
cise parenchymal transection line with appropriate safety margins (fig. 2) [14].

The images generated were then used for AR guidance. By overlaying images on the laparoscopic monitor, the surgeon can accurately see the surgical instruments, the tumour lesion, and the vascular structures. The main advantage of this approach is that it can compensate for real-time soft tissue deformation, which plays a major role in liver surgery and can be considered the main drawback of image-guided approaches relying on preoperatively acquired image datasets. The advantages of intraoperative CT scanning as an image guiding tool in comparison with IOUS image guidance are its ability to detect lesions located deep in the liver parenchyma and its objective reproducibility. Disadvantages encountered so far have been the lack of high-quality images of the liver parenchyma, as described by Kenngott et al. [14]. In practice, an integrated environment, as provided by hybrid operating rooms, is likely to prove necessary.

\section{Fluorescent Imaging and Liver Surgery}

With recent advances in intraoperative imaging technologies supplied in high-technology hybrid operating rooms, near-infrared (NIR) fluorescence imaging using indocyanine green (ICG) or methylene blue (MB) as contrast agents has experienced a rapid, renewed attention and expansion. The NIR fluorescence imaging techniques result from ICG or MB binding to plasma proteins emitting light with a peak emission wavelength of 800 or $830 \mathrm{~nm}$ when excited with light [15]. Fluorescence laparoscopy setups have been developed and are easier to apply than in open surgery, since a dark environment in the operating room is given. Here, we focus on possibilities to stain liver segments anatomically by NIR fluorescence imaging. This is of major importance against the background of the fact that anatomical liver segment resections have been shown to provide better oncological results than non-anatomical wedge resections [16, 17]. To date, identification of the segmental portal branches by IOUS is the standard for identifying liver segment anatomy. In laparoscopic liver surgery, IOUS is much more difficult to perform than in open surgery, and the results are hard to reproduce. As a matter of fact, only few specialised laparoscopic liver surgeons will be able to perform true anatomic liver segment resections under IOUS guidance. We believe that standardised procedures using NIR imaging of liver segments could lead surgeons to perform more accurate anatomic liver segment resections. Kokudo et al. [15] have described a technique by which ICG has to be injected into the portal vein branches while a Pringle manoeuvre is performed. With this technique, exact liver segment anatomy was identifiable in $94 \%$ of the patients. However, this presupposes the intraoperative identification of the segmental portal vein branch and therefore represents a proof of principle rather than a true technical advantage for most surgeons.

An interesting and more pragmatic approach within mod-

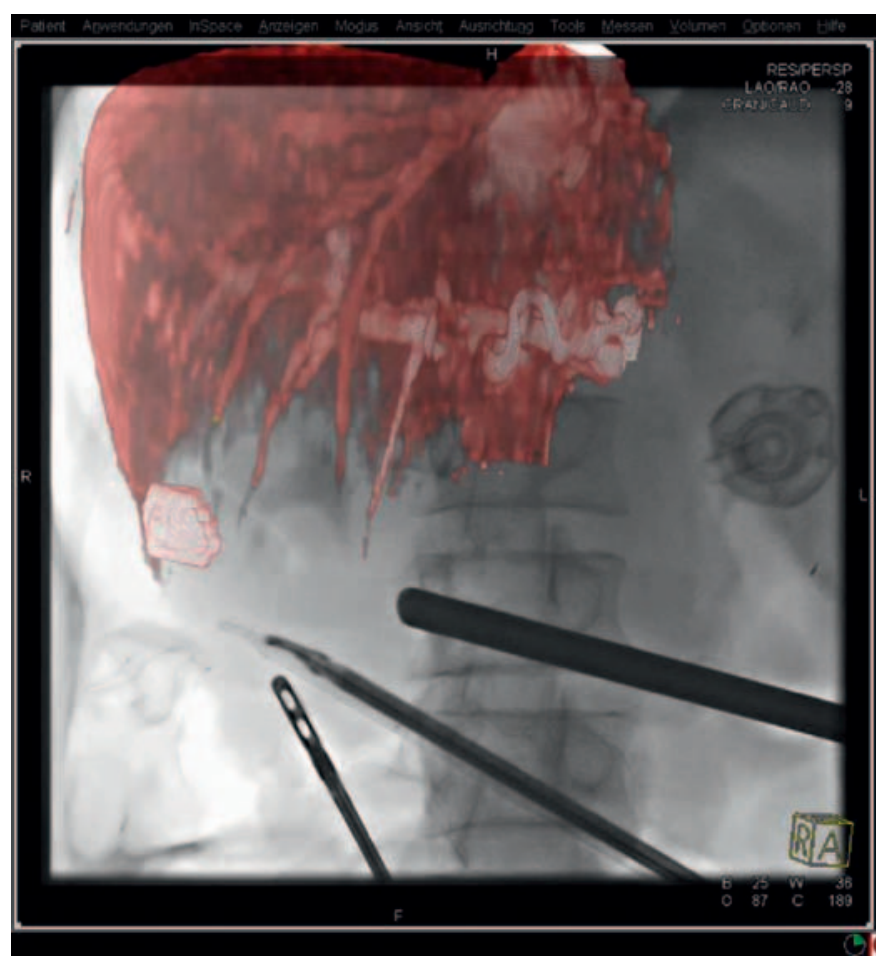

Fig. 2. Fluoroscopy with AR: The preoperative MRI data were superimposed on the real-time fluoroscopy, with the surgical instruments being clearly visible in relation to the tumor. Image courtesy of Siemens AG, Healthcare Sector, and Springer Science+Business Media.

ern hybrid operating rooms providing possibilities of modern angiography might be the insertion of ICG through highly selective catheterisation of liver segment arteries by means of a transfemoral technique. Liver segment visualisation could then be provided within a true interdisciplinary workflow between surgeons and radiologists. Most notably, the patient with a malignant liver lesion would benefit from an exact segmental liver resection, leading to better long-term survival.

\section{Conclusion}

Various image guidance techniques for pre- and intraoperative oncological liver surgery have been described. Reliable real-time image guidance is expected to become an essential technique in the future, particularly in laparoscopic liver surgery. From our point of view, AR guidance and fluorescent imaging in the setting of a modern hybrid operating theatre are promising approaches to achieve the balance between best oncological outcome and a surgical technique minimising harm to the patient in laparoscopic liver surgery.

\section{Disclosure Statement}

No conflicts of interest. 


\section{References}

1 Adam R, De Gramont A, Figueras J, Guthrie A, Kokudo N, Kunstlinger F, Loyer E, Poston G, Rougier P, Rubbia-Brandt L, Sobrero A, Tabernero J, Teh C, Van Cutsem E; Jean-Nicolas Vauthey of the EGOSLIM (Expert Group on OncoSurgery management of LIver Metastases) group: The oncosurgery approach to managing liver metastases from colorectal cancer: a multidisciplinary international consensus. Oncologist 2012;17:1225-1239.

2 Radtke A, Sotiropoulos GC, Molmenti EP, Schroeder T, Peitgen HO, Frilling A, Broering DC, Broelsch CE, Malago' M: Computer-assisted surgery planning for complex liver resections: when is it helpful? A single-center experience over an 8-year period. Ann Surg 2010;252:876-883.

$\checkmark 3$ Oldhafer KJ, Stavrou GA, Prause G, Peitgen HO, Lueth TC, Weber S: How to operate a liver tumor you cannot see. Langenbecks Arch Surg 2009; 394:489-494.

4 Chopra SS, Eisele RM, Denecke T, Stockmann M, Lange T, Eulenstein S, Schmidt SC, Neuhaus P: Advances in image guided conventional and minimal invasive liver surgery. Minerva Chir 2010; 65:463-478.

5 Kulemann V, Schima W, Tamandl D, Kaczirek K, Gruenberger T, Wrba F, Weber M, Ba-Ssalamah A: Preoperative detection of colorectal liver metastases in fatty liver: MDCT or MRI? Eur J Radiol 2011;79:e1-6.

6 Makuuchi M, Hasegawa H, Yamazaki S, Takayasu $\mathrm{K}$, Moriyama N: The use of operative ultrasound as an aid to liver resection in patients with hepatocellular carcinoma. World J Surg 1987;11:615-621.
Bonanni L, De'liguori Carino N, Deshpande R, Ammori BJ, Sherlock DJ, Valle JW, Tam E, O'Reilly DA: A comparison of diagnostic imaging modalities for colorectal liver metastases. Eur J Surg Oncol 2014;40:545-550.

8 Wildi SM, Gubler C, Hany T, Petrowsky H, Clavien PA, Jochum W, Gerlach T, Fried M, Mullhaupt B: Intraoperative sonography in patients with colorectal cancer and resectable liver metastases on preoperative FDG-PET-CT. J Clin Ultrasound 2008;36:20-26.

$\checkmark 9$ Kamiyama T, Tahara M, Nakanishi K, Yokoo H, Kamachi H, Kakisaka T, Tsuruga Y, Matsushita M, Todo S: Long-term outcome of laparoscopic hepatectomy in patients with hepatocellular carcinoma. Hepatogastroenterology 2014;61:405-409.

10 Luo LX, Yu ZY, Bai YN: Laparoscopic hepatectomy for liver metastases from colorectal cancer: a meta-analysis. J Laparoendosc Adv Surg Tech A 2014;24:213-222.

11 Montalti R, Berardi G, Laurent S, Sebastiani S, Ferdinande L, Libbrecht LJ, Smeets P, Brescia A, Rogiers X, de Hemptinne B, Geboes K, Troisi RI: Laparoscopic liver resection compared to open approach in patients with colorectal liver metastases improves further resectability: oncological outcomes of a case-control matched-pairs analysis. Eur J Surg Oncol 2014;40:536-544.

12 Twaij A, Pucher PH, Sodergren MH, Gall T, Darzi A, Jiao LR: Laparoscopic vs open approach to resection of hepatocellular carcinoma in patients with known cirrhosis: systematic review and metaanalysis. World J Gastroenterol 2014;20:8274-8281.
3 Buell JF, Cherqui D, Geller DA, et al.; World Consensus Conference on Laparoscopic Surgery: The international position on laparoscopic liver surgery: The Louisville Statement, 2008. Ann Surg 2009; 250:825-830.

14 Kenngott HG, Wagner M, Gondan M, Nickel F, Nolden M, Fetzer A, Weitz J, Fischer L, Speidel S, Meinzer HP, Böckler D, Büchler MW, MüllerStich BP: Real-time image guidance in laparoscopic liver surgery: first clinical experience with a guidance system based on intraoperative CT imaging. Surg Endosc 2014;28:933-940.

15 Kokudo N, Ishizawa T (eds): Fluorescent Imaging: Treatment of Hepatobiliary and Pancreatic Diseases. Front Gastrointest Res, vol 31. Basel, Karger, 2013 , pp 49-57.

16 Cucchetti A, Qiao GL, Cescon M, Li J, Xia Y, Ercolani G, Shen F, Pinna AD: Anatomic versus nonanatomic resection in cirrhotic patients with early hepatocellular carcinoma. Surgery 2014;155:512-521.

17 Cucchetti A, Cescon M, Ercolani G, Bigonzi E, Torzilli G, Pinna AD: A comprehensive meta-regression analysis on outcome of anatomic resection versus nonanatomic resection for hepatocellular carcinoma. Ann Surg Oncol 2012;19:3697-3705. 